\title{
Aktive Angehörige sind bei COPD ein Segen
}

\author{
COPD-Patienten, die mit einem körperlich aktiven Angehörigen zusammenleben, \\ bewegen sich auch selbst mehr. Das ist wichtig für das Therapiemanagement.
}

_ Für eine Querschnittstudie wurden 125 COPD-Patienten im Altern von $67 \pm 7$ Jahren mit einer $\mathrm{FEV}_{1}$ von $51 \pm 17 \%$ des Sollwerts rekrutiert. Sie trugen in ihrem häuslichen Umfeld für mindestens sieben Tage einen validierten Aktivitätsmonitor. Zur selben Zeit trug auch jeweils ein in derselben Wohnung lebender Familienangehöriger einen Monitor. Teilnehmer mit einem Energieverbrauch $<1,5$ metabolischen Äquivalenten (MET) wurden als inaktiv definiert, 1,5-3 MET galten als leichte, $>$ 3,0 MET als moderate bis intensive Aktivität. Per Fragebogen wurden die Bewegungsmotivation erfasst.

Die COPD-Patienten waren signifikant inaktiver als ihre Angehörigen, welche auch häufiger eine intrinsische Motivation zur Bewegung aufwiesen. Unabhängig von der Bewegungsmotivation zeigte sich aber, dass COPD-Patienten mehr Zeit mit moderater bis intensiverer körperlicher Aktivität verbrachten, wenn sie einen aktiveren Angehörigen hatten (18 vs. $31 \mathrm{~min} / \mathrm{d}, \mathrm{p}=0,002)$. Ein aktiverer Angehöriger steigerte die Wahrscheinlichkeit, dass der Patient selbst körperlich aktiv wurde, um das Vierfache.

- Mesquita R, Nakken N, Janssen DJA et al. Activity levels and exercise motivation in patients with COPD and their resident loved ones. Chest. 2017;151:1028-38

\section{KOMMENTAR}

Wieder einmal zeigt sich, welch erheblichen Beitrag Familienangehörige leisten können. Sie sollten idealerweise in das Therapiemanagement von COPD-Patienten einbezogen werden. Die Familie kann auch z. B. bei der planmäßigen Verwendung von Medikamenten und Sprays oder bei der Inhalationstechnik von großer Hilfe sein.

Die Studienautoren diskutieren weitere Ansätze. Bezieht man die Angehörigen etwa im Rahmen einer pneumologischen Rehabilitation ein, so hat dies einen positiven Effekt auf die CopingStrategien. Angehörige können die Patienten zudem im Alltag motivieren, indem sie sie etwa zu gemeinsamen tägli-

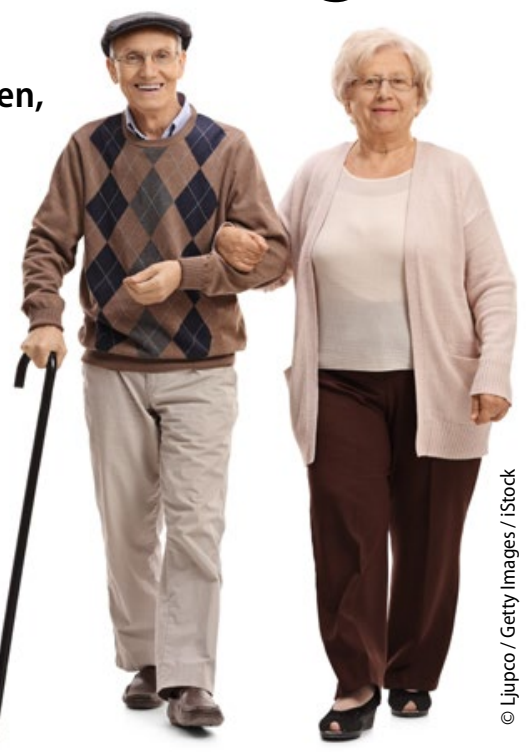

Ohne sie würde er gar nicht rausgehen.

chen Spaziergängen anhalten oder mehr in den Haushalt einbinden. Umgekehrt gilt, dass sie sie mit ungünstigem Gesundheitsverhalten auch negativ beeinflussen - etwa wenn sie weiter rauchen.

Leider waren 85\% der Studienteilnehmer in den GOLD-Stadien II und III, was die Aussagekraft für die "Rand-Stadien" I und IV schmälert.

Dr. phil. R. Glöckl

Hier steht eine Anzeige.

\author{
Springer
}

